\title{
Focal Point Detection Based on Half Concentric Lens Model for Singular Point Extraction in Fingerprint
}

\author{
Natthawat Boonchaiseree and Vutipong Areekul \\ Kasetsart Signal \& Image Processing Laboratory (KSIP Lab), \\ Department of Electrical Engineering, Kasetsart University, Bangkok, 10900, Thailand \\ g4965124@ku.ac.th, vutipong.a@ku.ac.th
}

\begin{abstract}
A focal point is a kind of singular points, closely related to a core point, which can be derived from curvature of fingerprint ridges and valleys. It is expected that the focal point is more reliable than the core point in case of low quality fingerprint. This paper proposes a new efficient focal point localization method based on a half concentric lens model. The half concentric lens window, with directional adaptive, accelerates convergence of a focal point localization process rapidly. Moreover, concentric lens similarity factor is introduced in order to measure orientation and stability of an extracted focal point. From experimental results, the proposed scheme is out-performed most of singular point detection schemes in literature in term of location accuracy and consistency. For computational complexity, algorithm requires average 75 millisecond execution-time from original fingerprint to a unique focal point.
\end{abstract}

Keywords: Singular Point, Focal Point, Half Concentric Lens, Concentric Lens's Similarity, Fingerprint Registration.

\section{Introduction}

Large scale fingerprint identification requires extremely fast and efficient algorithm in order to satisfy practical demands such as national identification. To achieve this goal, algorithm should capture strong and distinguished global features. Consider pivotal features existing in general fingerprint, singular points are some of these global features which are easily noticed by human eyes. In general, singular point can be classified into two types; core point and delta point. A focal point [1], closely related to a core point, can be included as one of a kind of singular points.

In practical fingerprint identification, singular points are not easily detected. There exist many problems caused by finger conditions and live-scan fingerprint sensors. For example, finger conditions can be too dry or too wet resulting in fingerprints with low quality. It becomes worse if dust, sweat, and old prints are included. Moreover, different fingerprint sensor types can cause different types of noises and quality. Somehow, size of sensor is matter due to where people press their fingers, resulting in partial fingerprint with missing singular point. On the other hand, arch type of fingerprint has no core and most algorithms have run into the difficulties of localizing its core. Finally, non-linear distortion and elastic distortion is occurred between finger 
and sensors with various pressures. These problems drastically reduce accuracy of most singular point detection schemes reported in literature.

Existing works on locating singular points can be classified into three broad approaches; pattern-based, partitioning-based, and projection-based methods. The pattern-based method employs fixed patterns such as core and delta models. Then these patterns are correlated with orientation fields or ridges of fingerprint in order to find the locations of similar patterns. For example, Kawagoe and Tojo proposed the classic and popular method, called Poincare' index in 1984 [2]. This method searches for fixed patterns of core, delta, and whorl in fingerprint. A successful improvement of this classic method from Bazen and Gerez can be found in [3]. Park et.al. [4] analyzed directional field pattern and proposed filtering rules to detect singular point including arch type. Another sub-classes of pattern-based approach is called complex symmetrical filters, introduced by Nilsson and Bigun [5]. These filter shapes are similar to core and delta. Singular point detection is obtained by convolving fingerprint orientation fields with these filter models. Hence computational complexity depends on sizes of filters and fingerprint image.

The second approach, called partitioning based method, utilize boundary of quantized directional fields which leads to singular point. For example, Hung and Huang [6], introduced fault-line, a borderline which separate two adjacent regions of the same quantized directional fields. These fault-lines converge to singularities. Another example, by Ramo et al. [7], used transition lines of orientation fields and detected reference points by inspection of line intersections.

The third approach is referred to as projection-based method. The idea is to project fingerprint information, such as orientation fields, into some mathematic models or patterns, and analyze these models and patterns according to a priori knowledge, resulting in singular point position. For example, Jiang et al. introduced algorithm based on hierarchical analysis of the orientation coherence [8]. Liu et al. proposed a new way to project orientation fields into another field called local axial symmetry fields, then these fields is used to find a reference point location [9]. By projectionbased approach, the focal point was first introduced by Rerkrai and Areekul [10]. The focal point is a centroid of crossings, the intersection of two normal lines or gradients of curved ridges. The original focal point algorithm was demonstrated experimentally to be quite stable and a proof of concept, but it was of a very high computational complexity. Later Areekul et al. [11] introduced the second algorithm. Instead of calculating all enormous crossings to find a reliable centroid, this algorithm employs mean-shift approach by allowing iterative search along highly curved ridges. This approach reduces amount of computational time into practical implementation. However, this algorithm still needs fingerprint enhancement in order to effectively track local ridges. Recently Areekul and Boonchaiseree [12] introduced the third algorithm by analyzing a focal point from orientation fields instead of true ridges in order to reduce computational complexity without losing accuracy. In addition, this previous algorithm introduced a fixed half circle window which is suitably designed for orientation field selection, resulted in algorithm having a good performance.

In this paper, the concept of a half concentric lens or a half circle window from [12] is extended with directional adaptation. Moreover, concentric lens similarity factor is introduced in order to control algorithm's convergence. 


\section{Concentric Lens Similarity Factor}

This proposed algorithm allows a half concentric lens to be rotated in order to find suitable directional field's curves in each iteration step. The half concentric lens or a geometric half circle window, with radius of $R$ (pixels) and a center point at $\left(x_{c}, y_{c}\right)$ (pixels), contains some number of partitioning blocks as shown in Fig. 1(a). Note that all blocks with partial parts inside this half circle window are included.

In order to measure pattern of directional fields in a half circle window or a half concentric lens, we define perfect directional fields in form of a concentric pattern as shown in Fig 1(d). Then we define concentric lens similarity factor (CLS), $\alpha$, as following equation.

$$
\alpha=\frac{N}{M}\left(1-\sum_{(i, j) \in C L}^{N} \frac{\left|\theta_{f h}(i, j)-\theta_{c l}(i, j)\right|}{N \pi / 2}\right)
$$

where $\theta_{f h}(i, j)$ and $\theta_{c l}(i, j)$ are actual DFs in half circle window and ideal DFs of perfect concentric lens pattern, respectively. Each DF of block $i^{\text {th }}$ column and $j^{\text {th }}$ row should be member of concentric lens, $C L$, or in area of half circle window. $N$ is a number of reliable DF (discussed in 3.1) and $M$ is total number of DF in half circle window. Note that CLS factor ranges from zero to one where one the most similarity and zero the least similarity. Fig 1 (b,c,d) shows CLS factor with various DF patterns.

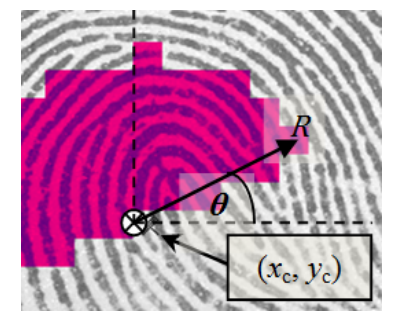

(a) Half Concentric Lens

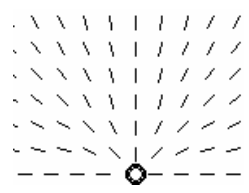

(b) $\alpha=0$

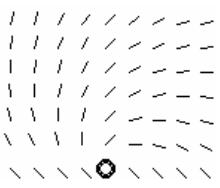

(c) $\alpha=0.5$

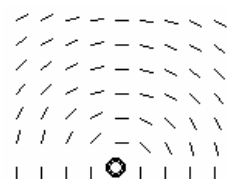

(d) $\alpha=1$

Fig. 1. (a) Half Concentric Lens or Geometric Half Circle Window. (b), (c) and (d) Concentric Lens Similarity Factor (CLS) with various DF patterns.

\section{Focal Point Extraction Algorithm}

The proposed focal point detection algorithm consists of the following steps: preprocessing, initial point localization, intermediate centroid iterative, and focal point selection. These steps are explained as follows.

\subsection{Preprocessing}

The input fingerprint image is partitioned into $N \times N$ blocks ( $N=16$ in this experiment), and its directional field of each block is carefully calculated by using Hong's method [13]. For higher precision of directional field estimation at pixel resolution, Bazen and 
Gerez's approach [3] is more suitable. The $\varphi_{f}(i, j)$ is a gradient, perpendicular to its directional field $\theta_{f}(i, j)$ in the same block with position $(i, j)$. Then $\operatorname{Coh}(i, j)$, coherence of directional field, is calculated using method in [3]. The $\operatorname{Coh}(i, j)$ is employed as a strength of directional field in each block, ranging from zero to one $(0,1)$. This $\operatorname{Coh}(i, j)$ value is used to indicate reliability of each directional field. In this experiment, DF with $\operatorname{Coh}(i, j)>0.3$ consider reliable and use in localization process. The rests are ignored. Note that DF smoothing method is not applied in our scheme in order to preserve and protect original DFs as possible.

\subsection{Initial Point Localization}

Let $x_{B}(i, j)$ and $y_{B}(i, j)$ be a center of block coordinate at column $i^{\text {th }}$ and rows $j^{\text {th }}$, respectively. $\varphi_{f}(i, j)$ represents a gradient orientation or an orientation with perpendicular to its directional field. A linear equation through the center of block coordinate is

$$
y-y_{B}=m_{B}\left(x-x_{B}\right) ; \text { where } m_{B}=\tan \left(\varphi_{f}(i, j)\right)
$$

where $m_{B}$ is a slope of gradient orientation. Our goal is to find all crossings of these gradients. However, some parallel DFs or low curvature may spread gradient crossings far apart resulting in focal point's divergence. Hence a gradient orientation constraint between two DFs is defined as

$$
\Delta \varphi=\left|\varphi_{f 1}-\varphi_{f 2}\right|>\varphi_{\text {threshold }},
$$

where $\Delta \varphi$ is absolute gradient difference between gradient $\varphi_{f 1}$ and gradient $\varphi_{f 2}$. In order to obtain a crossing point from two gradients, the above constraint must be satisfied. In experiment, if $\Delta \varphi$ is less than $\varphi_{\text {threshold }}=30$ degree $(\pi / 6)$, then a gradient crossing point could be calculated. Assume that $\left(x_{P}, y_{P}\right)$ is a coordinate of the gradient crossing and it can be obtained by

$$
\left(x_{p}=\frac{m_{B 2} x_{B 2}-m_{B 1} x_{B 1}+y_{B 1}-y_{B 2}}{m_{B 2}-m_{B 1}}, y_{p}=\frac{m_{B 2}\left(y_{B 1}-m_{B 1} x_{B 1}\right)+m_{B 1}\left(y_{B 2}-m_{B 2} x_{B 2}\right)}{m_{B 2}-m_{B 1}}\right)
$$

To control quality of a gradient crossing point contributed to a focal point, another constraint, related to distance between crossing $\left(x_{P}, y_{P}\right)$ and center of block $\left(x_{B 1}, y_{B 1}\right)$ and $\left(x_{B 2}, y_{B 2}\right)$, is defined as

$$
\Delta d=\left|d_{B 1 C}-d_{B 2 C}\right|<\Delta d_{\text {threshold }}
$$

where $d_{B 1 C}$ and $d_{B 2 C}$ are distance between crossing $\left(x_{P}, y_{P}\right)$ and a center of block $\left(x_{B 1}\right.$, $\left.y_{B 1}\right)$ and $\left(x_{B 2}, y_{B 2}\right)$, respectively. This constraint helps filtering some overrated crossings, and $\Delta d_{\text {threshold }}$ is equal to 128 pixels ( 8 blocks) in this experiment.

Once all gradients crossings are calculated, the number of crossings in $N \times N$ blocks (16×16 in our experiment) is accumulated to create 2-D histogram of crossings as shown in Fig 2 (c). Then a center of the maximum block is a candidate for an initial point. Note that a number of initial points can be more than one in order to capture all possible local maximum points. 


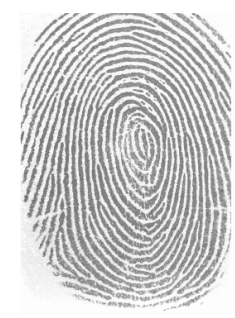

(a) Original

(b) Gradient Crossings

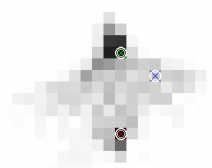

(c) Crossing Histogram

Fig. 2. Demonstrate Detection of Initial Point Localization in Crossing Histogram

\subsection{Intermediate Centroid Iterative}

This iterative process is a key of focal point localization and it composes of three major steps. Details of each step are discussed as follows.

\section{Step 1: Find Orientation with Maximum CLS}

Given initial point or an initial block, a half concentric lens window is located with radius $R$ blocks ( 6 blocks in this experiment). This window is rotated by 22.5 degree step size, with maximum range \pm 45 degree, resulting in only 5 orientations as shown in Fig 3. Then concentric lens similarity factor is calculated for each step size. If the initial point is outside image with non-overlapped area between concentric lens window and image, iteration will stop.

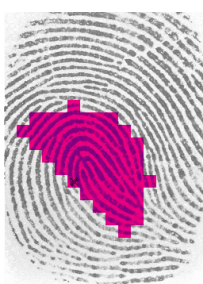

(a) $\alpha=0.6597$

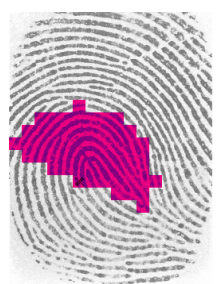

(b) $\alpha=0.7626$

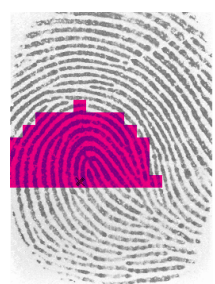

(c) $\alpha=0.8185$

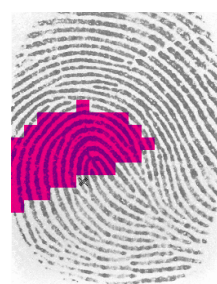

(d) $\alpha=0.8278$

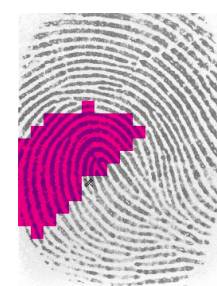

(e) $\alpha=0.8069$

Fig. 3. Half Concentric Lens in various Orientations $-\pi / 4,-\pi / 8,0, \pi / 8$, and $-\pi / 4$, respectively

\section{Step 2: Find Intermediate Centroid}

In this step, all gradient crossings by equations (2) to (5), generated from concentric lens window, are calculated. Then an current intermediate centroid, $\left(x_{C(k)}, y_{C(k)}\right)$, at $k^{\text {th }}$ iterative, can be derived by

$$
\left(x_{C(k)}, y_{C(k)}\right)=\left(\frac{1}{N(k)} \sum_{i=1}^{N(k)} x_{p(i, k)}, \frac{1}{N(k)} \sum_{i=1}^{N(k)} y_{p(i, k)}\right)
$$

where $N(k)$ is the total number of gradient crossings $\left(x_{P}, y_{P}\right)$, created in this $k^{\text {th }}$ iteration. 


\section{Step 3: Check Convergence}

Focal point converges when consecutive intermediate centroids do not move in iterative process. The $\varepsilon$ distance error and focal point convergence condition can be written by

$$
\varepsilon=\sqrt{\left(x_{C(k)}-x_{C(k-1)}\right)^{2}+\left(y_{C(k)}-y_{C(k-1)}\right)^{2}}<\varepsilon_{\text {Threshold }}
$$

To be precise, if distance error between a current intermediate centroid $\left(x_{C(k)}, y_{C(k)}\right)$ and a previous intermediate centroid $\left(x_{C(k-1)}, y_{C(k-1)}\right)$ is less than $\varepsilon_{\text {Threshold }}$, this iterative process will be terminated. Otherwise, the current intermediate centroid is a new initial point and iterative process continues (go back to Step 1 again). Note that iterative process has limited number of iterations to 10 rounds in order to protect limit cycle situation. The $\varepsilon_{\text {Threshold }}$ is set to zero (pixel) in this experiment.

\subsection{Focal Point Selection}

In general, if convergence condition is satisfied, the focal point is selected from the last intermediate centroid of the last iteration. Moreover, step 1 is employed again to find maximum CLS factor $\alpha$ which represented the best orientation for the detected focal point. Overall flowchart of the new focal point localization algorithm is concluded in Fig 4.

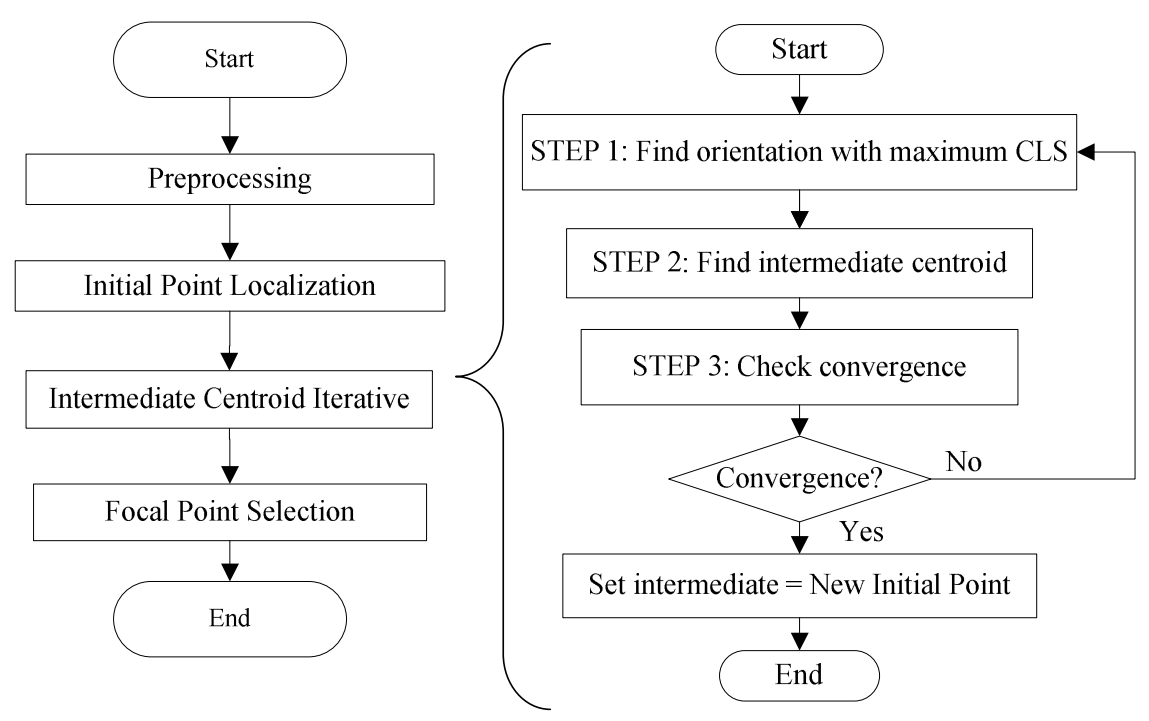

Fig. 4. Flowchart of the Proposed Focal Point Localization Algorithm

\section{Focal Point's Error Measurement and Evaluation}

Evaluation and error measurement of a focal point must be carefully designed and tested because the focal point cannot be visually detected by human eyes then it cannot be manually marked. Two approaches are proposed for distance error measurement. Then orientation error measurement is also concluded. 


\subsection{Distance Error Measurement}

In general, most singular point detection algorithms compare location of detected singular points with manually marked cores and deltas. Unfortunately, a pinpoint location of a focal point cannot be manually located because of invisible location. However, an expected area can be estimated. Therefore, in [12], the method "A" is introduced by linearly projecting an obtained focal point into another fingerprint image with 3 manually reference points. The second method " $\mathrm{B}$ " manually projects all obtained focal points into one fingerprint image. Both methods are discussed in detail as follows.

\section{a) Evaluation Method A: Approximately Linear Projection Method [12]}

In this evaluation method, three reference points are manually marked around singularity area of each finger, 8 fingerprint images, of all 800 images in FVC2000 Db2a database. Then linear projection can be applied to relocate an obtained focal point from one image to another image. A location of the focal point is projected onto two sides of a triangle, formed by 3 manually marked points [12]. By keeping the same projection ratio of triangle sides, the projected focal point can be relocated from one image to another image, and distance error can be measured. In Facts, there are three possible projection ways by selecting two out of three sides of triangle combinations. All possible ways are used and error distance is averaged. Detail is discussed in [12].

This method suffered from two major causes; linear projection error and manually 3-points marked error. Unfortunately, linear projection is not good estimation because of nonlinear distortion of fingerprint. Moreover, distance error depends on three marked points. If a focal point is inside a marked triangle, distance error is reasonable. However, if the focal point is outside the marked triangle, error is not reasonable. This method is very useful in case of computerized evaluation for optimizing parameters. However, experimental results may not be reliable to compare with other's results.

\section{b) Evaluation Method B: Manually Marked Projection Method}

To solve elastic problems in Method A, for each finger with 8 fingerprint images, other seven focal points are manually marked into one selected fingerprint image. Then distance error can be calculated by averaging distance error between the reference focal point to other 7 focal points. All focal points are rotated to be the reference focal point, resulted in distance error for each reference focal point. Finally, distance error from 8 reference points is averaged, resulted in average distance error for this finger. Clearly this method should result less error than Method A because nonlinear projection error is excluded. Only manually marked error may exist.

\subsection{Orientation Error Measurement}

Orientation error, $O E$, can be measured by using Liu's method [8] is defined as

$$
O E=\min \left\{\left|\theta_{P}-\theta_{R}\right|, \pi-\left|\theta_{P}-\theta_{R}\right|\right\}, \quad-\frac{\pi}{2}<\theta_{P}, \theta_{R}<\frac{\pi}{2}
$$

where $\theta_{P}$ is manually measured orientation from 3 marked points, and $\theta_{R}$ is extracted orientation, referred to the focal point. (The total result of $O E$ will be quantized by 5 orientations with range \pm 45 degree for evaluation in this experiment.). 


\section{Experimental Results}

Experimental results tested on FVC2000Db2A (100 fingers, 8 fingerprints /finger, capacitive sensor size $364 \times 256$ pixels, $500 \mathrm{dpi}$ ) which is also tested in $[4,5,6,7,10,11]$. Accuracy performance comparisons of various algorithms, referred to distance error and orientation error, are shown in Table 1 and Table 2, respectively. Computational complexity in term of execution time can be compared among limited algorithms using Intel Centrino Duo, CPU 1.6GHz, and 2MBs of RAM, as shown in Table 3.

Table 1. Position Accuracy Comparison of Singular Point Detection Algorithms in each Literature on FVC2000Db2A Database

\begin{tabular}{|c|c|c|c|c|c|c|c|}
\hline \multirow[b]{2}{*}{ Algorithms } & \multicolumn{5}{|c|}{ Distance Error, $D E$ (pixels) } & \multicolumn{2}{|c|}{ Accuracy (\%) } \\
\hline & {$[0,10]$} & $(10,20]$ & $(20,40]$ & $>40$ & Fail & $\begin{array}{l}\leq 10 \\
\text { pixel }\end{array}$ & $\begin{array}{l}\leq 20 \\
\text { pixel }\end{array}$ \\
\hline Proposed Alg./Eval.B & 668 & 101 & 26 & 5 & - & 83.50 & 96.12 \\
\hline M. Liu et.al. [8] & 659 & 90 & 25 & 13 & 13 & 82.37 & 93.62 \\
\hline T. Liu et.al. [9] & 654 & 91 & $\leftarrow 46$ & $\longrightarrow$ & 9 & 81.75 & 93.12 \\
\hline Proposed Alg./Eval.A & 624 & 143 & 20 & 13 & - & 78.00 & 95.87 \\
\hline Areekul [12]/Eval.B & 610 & 124 & 44 & 21 & 1 & 76.25 & 91.75 \\
\hline \multirow{2}{*}{ Algorithm } & \multicolumn{5}{|c|}{ Distance Error, $D E$ (pixels) } & \multirow{2}{*}{\multicolumn{2}{|c|}{$\begin{array}{l}\text { Accuracy }(\%) \\
<15 \text { pixels }\end{array}$}} \\
\hline & \multicolumn{2}{|c|}{$[0,15)$} & \multicolumn{3}{|c|}{$>15$} & & \\
\hline Proposed Alg./Eval.B & & \multicolumn{3}{|c|}{49} & \multicolumn{2}{|c|}{93.875} \\
\hline K. Nilsson et. al. [5] & \multicolumn{2}{|c|}{749} & \multicolumn{3}{|c|}{51} & \multicolumn{2}{|c|}{93.625} \\
\hline Proposed Alg./Eval.A & & & & 70 & & \multicolumn{2}{|c|}{91.250} \\
\hline \multirow{2}{*}{ Algorithm } & \multicolumn{7}{|c|}{ Magnitude Standard Deviation (pixels) } \\
\hline & \multicolumn{4}{|c|}{ All FVC2000 Db2a Database } & h-typ & nly (144 & chs in *) \\
\hline C.H. Park et.al. [4] & & 6.385 & & \multicolumn{4}{|c|}{10.690} \\
\hline *Proposed Alg./Eval.B & \multicolumn{4}{|c|}{$7.301($ Means $=6.481)$} & \multicolumn{3}{|c|}{$10.667($ Means $=11.885)$} \\
\hline *Proposed Alg./Eval.A & \multicolumn{4}{|c|}{$9.327($ Means $=10.532)$} & \multicolumn{3}{|c|}{$14.614($ Means $=14.412)$} \\
\hline
\end{tabular}

Table 2. Orientation Accuracy Comparison of Singular Point Detection Algorithms on FVC2000Db2A Database

\begin{tabular}{cccccc}
\hline \multirow{2}{*}{ Algorithm } & \multicolumn{3}{c}{ Orientation Error, OE (degree) } & \multicolumn{2}{c}{ Accuracy (\%) } \\
& {$[\mathbf{0 , 1 1 . 2 5}]$} & $(\mathbf{1 1 . 2 5 , 2 2 . 5}$ & $\mathbf{> 2 2 . 5}$ & Fail & $<\mathbf{2 2 . 5}$ pixels \\
\hline T. Liu. et.al. [9] & 690 & 47 & 50 & 13 & 93.647 \\
Proposed Alg. & 521 & 136 & 143 & - & 82.125 \\
\hline
\end{tabular}

Table 3. Compare Computational Time among Focal Point Localization Algorithms, FVC2000Db2A Database, with Intel Centrino Duo 1.6GHz and 2MBs of RAM

\begin{tabular}{lccc}
\hline & \multicolumn{3}{c}{ Average Execution Time (millisecond) } \\
Process & Algorithm in [11] & Algorithm in [12] & Proposed Algorithm \\
\hline Segmentation & 26.98 & - & - \\
DF Extraction & 13.10 & 13.10 & 13.10 \\
Enhancement & 535.72 & - & - \\
Focal Point Localization & 30.10 & 29.52 & 61.94 \\
Total & $\mathbf{6 0 5 . 9 0}$ & $\mathbf{4 2 . 6 2}$ & $\mathbf{7 5 . 0 4}$ \\
\hline
\end{tabular}


In Table 1, our proposed scheme with measurement method B yields the best experimental results in singular point localization accuracy. However, Table 2 shows problem with orientation accuracy of detected reference point. The main reason is that orientation is quantized into 5 steps with $\pi / 4$ apart. Hence accuracy may not be good but it can be improved in the near future. Finally, in Table 3, the proposed algorithm is compared with two previous algorithms in term of execution time.

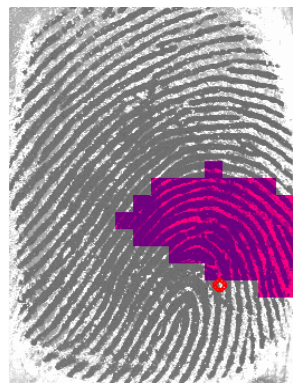

(a) \#38_1

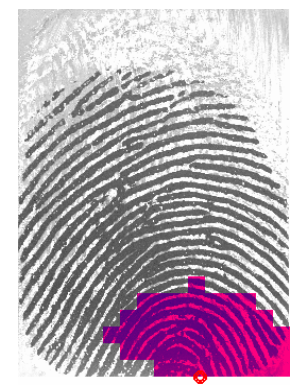

(b) \#38_4

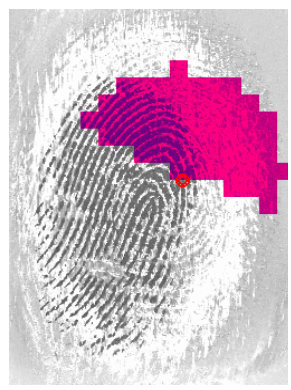

(c) \#81_7

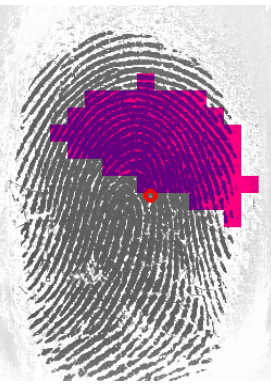

(d) \#81_8

$(D E=2.06$ pixels $)$

$(D E=9.53$ pixels $)$

$(D E=10.72$ pixels $)$

$(D E=2.07$ pixels $)$

Fig. 5. Good Results from Proposed Algorithm on FVC2000Db2A (DE by Method B Eval)

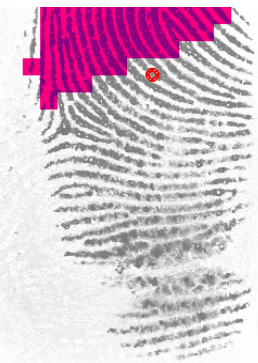

(a) \#22_1

$(D E=60.64$ pixels $)$

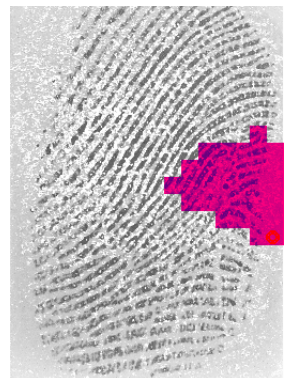

(b) \#69_4

$(D E=43.55$ pixels $)$

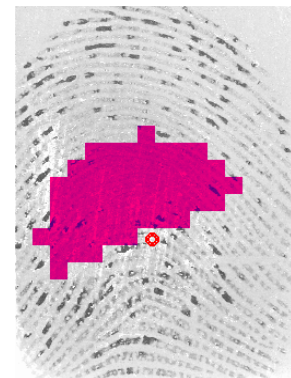

(c) \#100_1

$(D E=90.93$ pixels $)$

Fig. 6. Failure from the Proposed Algorithm on FVC2000Db2A (DE by Method B Eval)

Fig 5 shows good results from the proposed algorithm. Fig 6 reported failure from the proposed algorithm. For example, without most-north curve area in Fig 6(a), without right-side curve area in Fig 6(b), and low quality core in Fig 6 (c).

\section{Conclusion and Future Research}

In this paper, projection-base method (Focal Point Projection Concept) and templatebase method (Concentric Lens Model) are combined for a new proposed algorithm. The proposed algorithm provides fast convergence with high accuracy and good 
consistency, comparing with other singular point detection methods in literature. In the near future, applications of focal point could be proposed for fingerprint classification. The proposed algorithm can be further improved by multi-resolution approach. In addition, orientation accuracy must be improved and tested with other FVC databases such as thermal slide sensor or FVC2004 with high elastic distortion.

Acknowledgments. This work was partially supported by Department of Electrical Engineering, Kasetsart University, the Thailand Research Fund and the Commission on Higher Education through Grant No. RMU4980027, and JON KEE's Scholarship.

\section{References}

1. Maltoni, D., Maio, D., Jain, A.K., Prabhakar, S.: Handbook of Fingerprint Recognition. Springer, Heidelberg (2003)

2. Kawagoe, M., Tojo, A.: Fingerprint Pattern Classification. Pattern Recognition 17, 295303 (1984)

3. Bazen, A.M., Gerez, S.H.: Systematic Methods for the Computation of the Directional Fields and Singular Points of Fingerprints. IEEE Trans. Pattern Anal. Machine Intell. 24, 905-919 (2002)

4. Park, C.H., Lee, J.J., Smith, M.T.J., Park, K.H.: Singular Point Detection by Shape Analysis of Directional Fields in Fingerprint. Pattern Recognition 39, 839-855 (2005)

5. Nilsson, K., Bigun, J.: Registration of Fingerprints by Complex Filtering and by 1D Projections of Orientation Images. In: Kanade, T., Jain, A., Ratha, N.K. (eds.) AVBPA 2005. LNCS, vol. 3546, pp. 171-183. Springer, Heidelberg (2005)

6. Hung, D.C.D., Huang, C.: A Model Optimized Singular Point Detection Algorithm for Fingerprint Images. In: Proc. Florida Artificial Intelligence Research Symposium (9th), pp. 444-448 (1996)

7. Rämö, P., Tico, M., Onnia, V., Saarinen, J.: Optimized Singular Point Detection Algorithm for Fingerprint Images. In: Proc. Int'l. Conf. Image Processing, vol. 3, pp. 242-245 (2001)

8. Liu, M., Jiang, X.: Kot. A.C.: Fingerprint Reference-Point Detection. EURASIP Journal on Applied Signal Processing, 498-509 (2005)

9. Liu, T., Zhang, C., Hao, P.: Fingerprint Reference Point Detection Based on Local Axial Symmetry. In: Proc. Int'l. Conf. Pattern Recognition, vol. I, pp. 1050-1053 (2006)

10. Rerkrai, K., Areekul, V.: A New Reference Point for Fingerprint Recognition. In: Proc. Int'l. Conf. Image Processing, vol. 2, pp. 499-502 (2000)

11. Areekul, V., Suppasriwasuseth, K., Jirachaweng, S.: The New Focal Point Localization Algorithm for Fingerprint Registration. In: Proc. Int'l. Conf. Pattern Recognition, vol. IV, pp. 497-500 (2006)

12. Areekul, V., Boonchaiseree, N.: Fast Focal Point Localization Algorithm for Fingerprint Registration. In: Proc. 3rd IEEE ICIEA, pp. 2089-2094 (2008)

13. Hong, L., Wang, Y., Jain, A.K.: Fingerprint Image Enhancement: Algorithm and Performance Evaluation. IEEE Trans. on Pattern Analysis and Machine Intelligence 20(8), 777 789 (1998) 